\title{
POPULATION STUDY ARTICLE Reduced brain mammillary body volumes and memory deficits in adolescents who have undergone the Fontan procedure
}

\author{
Cristina Cabrera-Mino ${ }^{1}$, Bhaswati Roy ${ }^{1}$, Mary A. Woo ${ }^{1}$, Sadhana Singh ${ }^{2}$, Stefanie Moye ${ }^{1}$, Nancy J. Halnon ${ }^{3}$, Alan B. Lewis ${ }^{4}$, \\ Rajesh Kumar ${ }^{2,5,6,7}$ and Nancy A. Pike ${ }^{1}$
}

\begin{abstract}
BACKGROUND: Adolescents with single ventricle heart disease (SVHD) who have undergone the Fontan procedure show cognitive/memory deficits. Mammillary bodies are key brain sites that regulate memory; however, their integrity in SVHD is unclear. We evaluated mammillary body (MB) volumes and their associations with cognitive/memory scores in SVHD and controls. METHODS: Brain MRI data were collected from 63 adolescents (25 SVHD; 38 controls) using a 3.0-Tesla MRI scanner. Cognition and memory were assessed using Montreal Cognitive Assessment (MoCA) and Wide Range Assessment of Memory and Learning 2. MB volumes were calculated and compared between groups (ANCOVA, covariates: age, sex, and total brain volume [TBV]). Partial correlations and linear regression were performed to examine associations between volumes and cognitive scores (covariates: age, sex, and TBV). RESULTS: SVHD group showed significantly lower MoCA and WRAML2 scores over controls. MB volumes were significantly reduced in SVHD over controls. After controlling for age, sex, and TBV, MB volumes correlated with MoCA and delayed memory recall scores in SVHD and controls.

CONCLUSION: Adolescents with SVHD show reduced MB volumes associated with cognitive/memory deficits. Potential mechanisms of volume losses may include developmental and/or hypoxic/ischemic-induced processes. Providers should screen for cognitive deficits and explore possible interventions to improve memory.
\end{abstract}

Pediatric Research (2020) 87:169-175; https://doi.org/10.1038/s41390-019-0569-3

\section{INTRODUCTION}

Adolescents with single ventricle heart disease (SVHD) require at least three-staged palliative surgeries before the age of 4 years, culminating with the Fontan procedure. Many SVHD children experience a variety of cognitive symptoms, including memory, attentional, and behavioral impairments (i.e., intellectual deficits; learning difficulties; pragmatic language; social interaction; visuospatial, motor, and executive function deficits) that persist into adulthood. ${ }^{1}$ With significant improvements in fetal detection, surgical strategies, and perioperative care, survival rates have improved in SVHD patients. ${ }^{2}$ However, neurodevelopmental/ cognitive outcomes have only modestly improved. ${ }^{3}$ Such deficits can limit academic achievement, lower quality of life, and ultimately diminish the ability for self-care. ${ }^{4,5}$

Approximately $60 \%$ of children with SVHD show brain injury, commonly reported as changes in white or gray matter or cerebral thromboembolic events. ${ }^{1,6}$ Cognitive deficits, in particular memory, have been reported in SVHD and other complex congenital heart disease (CHD). Brain changes have been assessed and documented in several memory regulatory areas, including the hippocampus and anterior thalamus, in SVHD. ${ }^{7}$ However, due to their small size and location adjacent to bony structures and cerebral spinal fluid (CSF), mammillary bodies (MBs) have not been assessed in SVHD.

In addition to other memory circuitry structures (hippocampus, anterior thalamus, and fornix fibers), MBs, located in the inferior aspect of the brain, are important regions associated with spatial and episodic memory. These bodies are divided into medial and lateral nuclei and consist solely of projection cells that receive both excitatory and inhibitory inputs, while delivering only excitatory outputs. MBs receive inputs from the hippocampus through fornix fibers and project to anterior thalamus and then cortical sites. Injury to the MBs has resulted in poor performance in spatial and working memory tasks in animal studies. ${ }^{8}$ Furthermore, $\mathrm{MB}$ injury has been linked to memory deficits in multiple conditions, such as obstructive sleep apnea, heart failure, congenital central hypoventilation syndrome, children with epilepsy, preterm infants, and Korsakoff's syndrome. ${ }^{9-12}$ However, $M B$ injury has not been reported in SVHD patients.

Therefore, the purpose of this study was to evaluate MB volumes in SVHD and controls using high-resolution T1-weighted magnetic resonance imaging (MRI) procedures and to correlate $\mathrm{MB}$ volumes with various cognitive/memory scores.

\section{MATERIAL AND METHODS}

Participants

This comparative and correlational study was approved by the Institutional Review Boards at the University of California Los Angeles (UCLA) and Children's Hospital Los Angeles (CHLA) prior to implementation. A total of 63 adolescents were enrolled

\footnotetext{
${ }^{1}$ UCLA School of Nursing, University of California Los Angeles, Los Angeles, CA, USA; ${ }^{2}$ Department of Anesthesiology, University of California Los Angeles, Los Angeles, CA, USA; ${ }^{3}$ Division of Pediatric Cardiology, University of California Los Angeles, Los Angeles, CA, USA; ${ }^{4}$ Division of Pediatric Cardiology, Children's Hospital Los Angeles, Los Angeles, CA, USA; ${ }^{5}$ Department of Radiological Sciences, University of California Los Angeles, Los Angeles, CA, USA; ${ }^{6}$ Department of Bioengineering, University of California Los Angeles, Los Angeles, CA, USA and ${ }^{7}$ Brain Research Institute, University of California Los Angeles, Los Angeles, CA, USA Correspondence: Nancy A. Pike (npike@sonnet.ucla.edu)
}

Received: 13 March 2019 Revised: 8 August 2019 Accepted: 16 August 2019

Published online: 9 September 2019 
170

(25 SVHD, [mean age $15.88 \pm 1.24$ years, 15 males]; 38 controls [mean age $15.97 \pm 1.10$ years, 19 males]). Adolescents (ages 14-18 years) with SVHD who have undergone the Fontan procedure were recruited via flyers or physician referrals from UCLA and CHLA Medical Centers. Healthy controls were recruited from local high schools, word of mouth, and from the surrounding Los Angeles community. Exclusion criteria for SVHD and controls were claustrophobia, non-removable metal (such as braces, pacemakers), previous head injury, severe developmental delay (e.g., cerebral palsy or severe hypoxic injury) precluding active study participation, diagnosis of depression, premature birth ( $<37$ weeks gestation), history of previous extracorporeal membrane oxygenation (ECMO) use, documented stroke, and cardiac arrest. In addition, control participants were screened and excluded for any chronic medical or psychiatric conditions. The SVHD participants were matched to a control for age ( \pm 1 year), sex, and ethnicity. All SVHD and control participants completed a demographic information form. Clinical data were collected from medical records of the SVHD patients. For all participants aged $<18$ years, parental permissions and subject assents were obtained, and written and informed consents were obtained from participants aged $\geq 18$ years before data collection.

Data collection instruments

Cognitive assessment. We used the Wide Range Assessment of Memory and Learning, Second Edition (WRAML2), which is a wellvalidated and reliable measure of various aspects of memory and learning in children and adults (e.g., 5-90 years of age). ${ }^{13}$ The WRAML2 consists of six subtests, involving verbal and visual memory and attention/concentration, which yields the General Memory Index (GMI) score, and working memory, verbal and visual memory recognition, which produce the General Recognition Index (GRI) scores (normal, $100 \pm 15 ; \leq 85$ considered mild-tomoderate memory impairment). The alpha reliabilities for the core subtests range from 0.85 to $0.94 .^{14}$ The WRAML2-derived GMI score has been widely used to assess memory in the adolescent population both with and without $\mathrm{CHD} .^{15}$

The Montreal Cognitive Assessment (MoCA) is an administered screener used to measure multiple domains of cognitive function, such as visuospatial, attention/concentration, executive function, language, delayed memory recall, and naming. The visuospatial and executive function tasks are written items (i.e., threedimensional cube, Watson Clock Drawing Task, and alternating Trail Making Task Part B), and the remaining are scored based on verbal response. ${ }^{16}$ The total MoCA score ranges from 0 to 30 , and a score $<26$ is considered abnormal. The MoCA has been validated in adolescents both with and without CHD and has a Cronbach's alpha of $0.8 .^{14,16}$

Socioeconomic status. Annual household income reflects socioeconomic status (SES) and is derived from each subject's residential postal zip code. SES was calculated for each participant from the American Community Survey data available on Population Studies Center, Institute for Social Research (https://www. census.gov/programs-surveys/acs/).

Magnetic resonance imaging. Brain images from SVHD patients and controls were acquired using a 3.0-Tesla MRI scanner (Siemens, Magnetom Prisma, Erlangen, Germany). Participants were placed in the supine position in the scanner with foam padding on both sides of their head to minimize movement. If movement artifact was visually identified on images, the scan was repeated. High-resolution T1-weighted images were collected using a magnetization-prepared rapid-acquisition gradient-echo (MPRAGE) sequence [repetition time $(T R)=2200 \mathrm{~ms}$; echo time $(T E)=2.4 \mathrm{~ms}$; inversion time $=900 \mathrm{~ms}$; flip angle $(F A)=9^{\circ}$; matrix size $320 \times 320$; field of view $(F O V)=230 \times 230 \mathrm{~mm}$; slice thickness $=0.9 \mathrm{~mm}$ ). Proton density $(\mathrm{PD})$ and T2-weighted images
$\left(\mathrm{TR}=10000 \mathrm{~ms} ; \mathrm{TE} 1,2=12,124 \mathrm{~ms} ; \mathrm{FA}=130^{\circ}\right)$ were collected with a dual-echo turbo spin-echo pulse sequence in the axial plane $(230 \times 230 \mathrm{~mm}$ FOV, $256 \times 256$ matrix size, $3.5 \mathrm{~mm}$ slice thickness, and no interslice gap). In addition, all MRls were formally interpreted by a pediatric neuroradiologist, who was blinded by group assignment, to visually assess for any serious brain structural injury.

MRI data processing. Brain images were preprocessed using the statistical parametric mapping (SPM12) package (Wellcome Department of Cognitive Neurology, London, UK; http://www.fil. ion.ucl.ac.uk/spm/) and MATLAB-based (The MathWorks Inc, Natick, MA) custom software.

Both high-resolution T1-weighted image volumes of each subject were realigned and averaged to improve signal-to-noise ratios. The averaged images were bias-corrected for any potential image signal intensity variation using the unified segmentation approach, reoriented into Montreal Neurological Institute (MNI) common space (rigid-body affine transformation), and resampled to voxel size $0.7 \times 0.7 \times 0.7 \mathrm{~mm}$.

Total brain volume (TBV) calculation. A unified segmentation approach was used to partition the reoriented T1-weighted images into gray matter, white matter, and CSF tissue types. ${ }^{17}$ The TBV volumes were computed using gray matter and white matter probability maps. Voxels with a probability value $>0.5$ for gray and white matter in each subject were counted and TBV values were calculated.

$M B$ tracings and volume quantification. Using the reoriented images, the $M B$ areas were oversampled to a resolution of $0.2 \times$ $0.2 \times 0.2 \mathrm{~mm}$ and manual region of interest (ROI) measures of the left and right structures were performed separately using MRIcron (McCausland Center for Brain Imaging, University of South Carolina, Columbia, SC). One investigator, blinded to subject group assignment, performed the left and right $\mathrm{MB}$ tracings in all participants, as described in our previous reports. ${ }^{9-11}$ Briefly, sagittal and coronal views were used to establish the brain midline and the medial borders of both bodies. Coronal views were used to determine the superior and inferior MB edges, and consecutive sagittal slices were used to outline the entire structure with coronal and axial views to confirm the borders. Voxels were counted from each ROI, and the MB volumes were calculated by summing all voxels and multiplying by voxel volume. Figure 1 shows the MB volume tracing method used.

Intra- and inter-tracing reliability analyses. Intra-tracer reliability for outlining MB structures was examined by re-outlining in eight randomly selected SVHD and eight control participants by the same investigator who outlined both left and right bodies in all SVHD and controls. A second investigator, blinded to the first reviewer's results and subject group membership, traced MBs in the same participants (eight SVHD and eight controls), from which inter-tracing reliability was calculated. Inter- and intra-tracing reliabilities were calculated using intra-class correlation (ICC).

Statistical analysis. The Statistical Package for the Social Sciences software (IBM SPSS Statistics for Windows, v24, IBM Corp., Armonk, N.Y., USA) was used for data analyses. Demographic and cognitive data of SVHD and controls were evaluated using independent samples (two-tailed) $t$ tests for normally distributed data, Mann-Whitney $U$ test for non-normally distributed data, and Chi-square for categorical variables. The normality of each variable for SVHD and control subjects were evaluated using Shapiro-Wilk test. The MB volumes between SVHD and controls were assessed using analysis of covariance, with covariates of age, sex, SES, and TBV. Correlations between cognitive scores and MB volumes in combination SVHD and controls were assessed using partial 


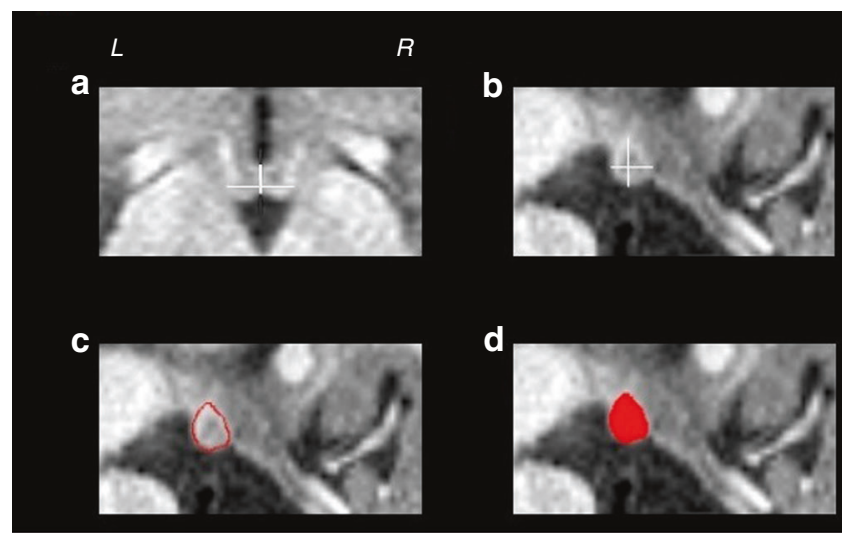

Fig. 1 Tracing method used to calculate mammillary body volumes. Midline borders for the left and right mammillary bodies were determined using coronal (a) views; these views also determine the superior and inferior right mammillary body edges (b) and consecutive sagittal slices were used to outline the entire structure in red (c) with coronal and axial views used to confirm the border. The right mammillary body is filled in red (d). The total volume of each mammillary body was calculated by counting the number of voxels in the traced red area

correlations (covariates: age, sex, and TBV). Linear regression analyses were conducted to examine possible confounding factors contributing to MB volume reduction (age, sex, and TBV as independent variables, and cognitive scores as dependent variables). All tests were two-tailed, with $p<0.05$ considered statistically significant.

\section{RESULTS}

Demographic, clinical, and cognitive characteristics

Demographic and cognitive scores from SVHD and control participants are summarized in Table 1 . No statistically significant differences appeared in age, sex, ethnicity, body mass index, or handedness between groups. However, SES was significantly lower in the SVHD group compared to that in controls.

Clinical characteristics of SVHD patients are listed in Table 1. The majority had a diagnosis of single right ventricle (67\%) and had an extracardiac Fontan (78\%). Many had a Fontan fenestration (26\%) and/or residual cyanosis (26\%) defined as oxygen saturations $<93 \%$.

The total MoCA score and all subscales, except for naming and orientation, were significantly lower in SVHD compared to that in controls $(p<0.04)$. In addition, WRAML2-derived GMI and GRI scores were significantly reduced, as well as subscores, including verbal and visual memory, attention/concentration, working memory, and visual and verbal recognition, in SVHD compared to that in controls $(p<0.006$; Table 1$)$. In addition, the mean TBV was significantly smaller in the SVHD over controls $(1.13 \pm 0.10$ vs. $1.24 \pm 0.10 \mathrm{~L} ; p<0.001$; covariates: age and sex).

Brain MRI findings

Abnormal brain MRI findings appeared in 8 SVHD subjects out of $25(32 \%)$ compared to 2 out of $38(5 \%)$ in the control group. Cerebral lesions were detected in 5 out of $25(20 \%)$ in the SVHD group, which consist of white mater changes, old infarctions/ strokes (incidental finding in all participants), and periventricular volume loss. Incidental developmental abnormalities in both the SVHD and control groups consisted of Rathke's cleft, pterygoid, or perivascular region cysts.

Tracing reliability

Intra-tracer reliability was high for MB tracings (ICC $=0.99, p<$ $0.001,95 \%$ confidence interval), indicating consistent tracings across all participants. Inter-tracing reliability for $\mathrm{MBs}$ was in agreement between the two investigators (left $\mathrm{MB}, \mathrm{ICC}=0.98$, $p<0.001$; right $\mathrm{MB}, \mathrm{ICC}=0.97, p<0.001,95 \%$ confidence interval).

$\mathrm{MB}$ volumes

An example of the MBs in a SVHD and age- and sex-matched control is shown in Fig. 2, and scatterplot with mean left and right mammillary volumes between groups is presented in Fig. 3. Both left and right MBs showed reduced volume in SVHD compared to that in controls (left, $40.24 \pm 19.18$ vs. $53.59 \pm 18.68 \mathrm{~mm}^{3}, p=0.01$; right, $42.57 \pm 17.26$ vs. $52.87 \pm 16.81 \mathrm{~mm}^{3}, p=0.03$; covariates: age, sex, and TBV). Both left and right MB volumes were reduced in SVHD subjects with identified cerebral lesions $(n=5)$ compared to participants without, but reductions were not statistically significant (left, $33.76 \pm 18.48$ vs. $37.51 \pm 17.43 \mathrm{~mm}^{3}, p=0.695$; right, $31.30 \pm 19.75$ vs. $40.12 \pm 18.62 \mathrm{~mm}^{3}, p=0.391$; covariates: age, sex, and TBV). MB volume differences continue after controlling for SES in addition to age, sex, and TBV.

Correlations between MB volumes and cognitive scores

Significant positive correlations appeared between the left MB volumes and WRAML2-derived verbal memory scores, and total MoCA and delayed memory recall subscores showed significant associations with left and right MB volumes in combined SVHD and control subjects (Table 2). Furthermore, linear regression analyses to predict MB volumes (separate left and right) were performed for each functional outcome (dependent variables) listed in Table 3. Both left and right $M B$ volumes were significantly associated with total MoCA, and subscale delayed memory recall and these associations were independent of age, sex, and TBV (Table 3). The GMI subscale verbal memory index correlates with the left $M B$ volume and visuospatial/executive function with the right $M B$ volume independent of age, sex, and TBV (Table 3).

Risk factors

No clinical risk factors such as current oxygen saturation level, number of surgeries, ventricular type, and number of medications correlated with smaller MB volumes.

\section{DISCUSSION}

This study identified significantly reduced left and right $M B$ volumes in SVHD compared to age- and sex-matched controls. Bilateral $M B$ volumes showed a significant association with cognitive scores, including memory deficits. The underlying etiology for reduced MB volume in SVHD is not completely known but may include delayed brain development, ${ }^{18}$ preoperative and postoperative brain injury associated with hypoxemia/ ischemia, reduced cerebral blood flow (CBF), hemodynamic instability, ${ }^{19,20}$ and possible nutritional deficiencies in the condition.

More recently, fetal and neonatal MRI studies have shown lower brain volumes (due to delayed brain maturation by up to 1 month) most commonly in the third trimester, possibly caused by reduced blood flow and cerebral oxygenation, ${ }^{21}$ with more defects on left ventricular outflow tract obstruction. ${ }^{18,20}$ Additionally, these infants may have lower birth weights, lower gestational ages, and smaller head circumferences, indicating brain developmental issues, compared to the general population. Brain immaturity in SVHD results in delays in myelination, placing increased risk for hypoxic-ischemic structural injury in the preoperative and postoperative period. ${ }^{22}$ Consequently, brain MRI studies have identified new or worsening white matter injury or other ischemic injury after neonatal cardiac surgery. ${ }^{22,23}$ As a highly vascular area of the brain, MBs are vulnerable to hypoxic injury.

MBs relay impulses from the amygdalae and hippocampi via the fiber projections of the mamillo-thalmic tract to the anterior 
Table 1. Demographic and cognitive scores of single ventricle heart disease and controls

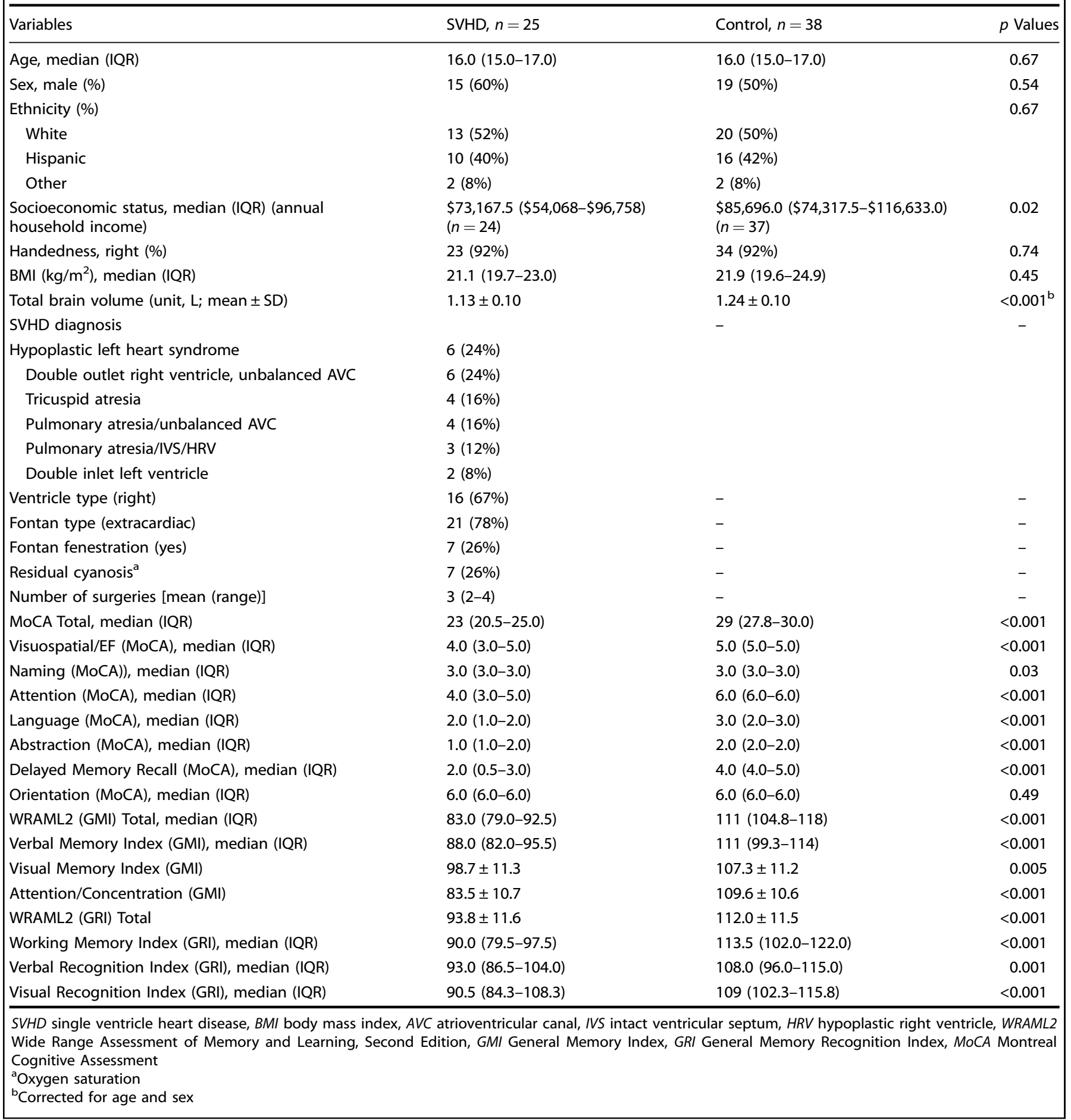

thalamus and may mediate several aspects of spatial and memory formation. ${ }^{24}$ A recent adolescent SVHD study found reduced gray matter volumes and tissue changes in multiple sites that are part of the mamillo-thalmic tracts and are associated with memory regulation (i.e., bilateral anterior, mid, and posterior thalamus; para hippocampus; and temporal gyrus). 6,14

In addition to the effects of surgery, SVHD patients are at risk of malnutrition due to inadequate nutritional intake, insufficient nutrient absorption secondary to decrease mesenteric perfusion, ${ }^{25}$ and increased metabolic demands that may all affect brain development. Furthermore, feeding difficulties are found to be a significant predictor of worse neurodevelopmental outcomes in infants with SVHD. ${ }^{26}$ Lower weight-for-age $z$ scores have been well documented after SVHD stage one and two palliation ${ }^{27}$ and most likely associated with the imbalance between pulmonary and systemic circulation. Although modest weight improvement is noted after the Fontan procedure, ${ }^{28}$ many remain underweight into adulthood. ${ }^{29}$ 


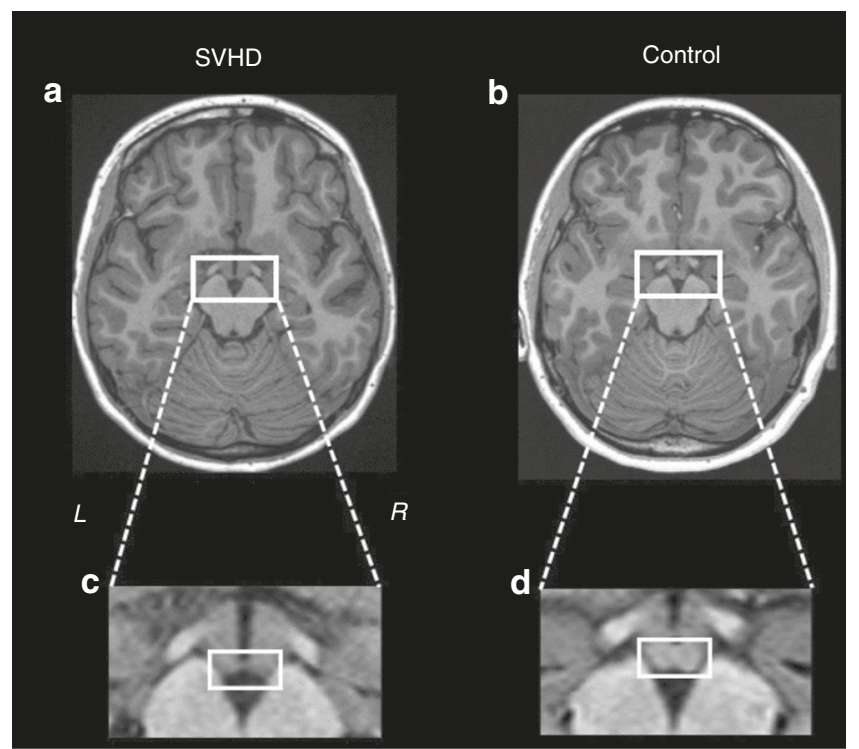

Fig. 2 High-resolution T1-weighted images show mammillary bodies in a SVHD (a) and control (b) subject (white rectangles). All images are in neurological convention ( $\mathrm{L}$ left, $\mathrm{R}$ right). Brain images (c, d) show magnified areas within the rectangle of the SVHD (a) and control (b) participants. The right and left mammillary bodies in the SVHD subject are almost absent compared to the control subject (c vs. d, white circles)

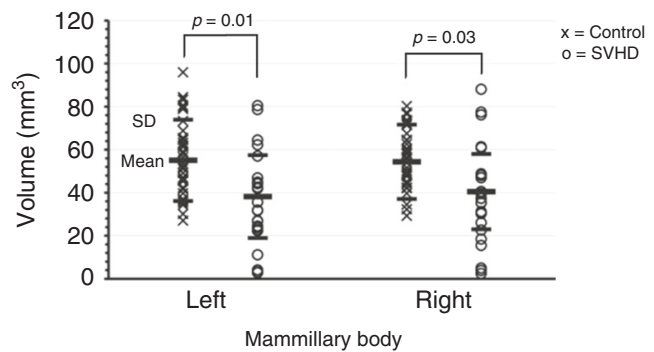

Fig. 3 Scatter plot shows smaller mean left (40.24 \pm 19.18 vs. 53.59 $\left.\pm 18.68 \mathrm{~mm}^{3}, p=0.01\right)$ and right $(42.57 \pm 17.26$ vs. $52.87 \pm 16.81$ $\mathrm{mm}^{3}, p=0.03$ ) mammillary body volumes in SVHD (O) vs. Controls (X), controlling for age, sex, and TBV
Nutritional deficits associated with MB volume loss has been seen in reduced vitamin $B$ intake or absorption, in particular thiamine. The volume loss and thiamine deficiency has been accompanied with anterograde memory loss in Korsakoff's syndrome, which is associated with chronic alcoholism. ${ }^{30}$ In animal models, thiamine deficiency has led to extensive damage to the MBs correlating with worse performance on memory and spatial tasks. ${ }^{31}$ In addition to dietary issues, thiamine deficiency can develop from use of diuretics or medical conditions associated with malabsorption such as anorexia, ${ }^{32}$ intestinal bypass, or the need for dialysis. ${ }^{33}$ One study in infants with ventricular septal defects and associated congestive heart failure treated with loop diuretics identified thiamine deficiency before and after surgical repair, despite adequate dietary intake of thiamine. ${ }^{34}$ Although we speculate that MB injury is associated with hypoxic-ischemic insult, early nutritional deficits commonly encountered in SVHD may contribute to inadequate neural protection or lead to additional neural injury.

Our findings identified lower SES in the SVHD group compared to that in controls, with $50 \%$ of the SVHD sample of Hispanic ethnicity. Disparities have been identified in population-based studies in children with critical CHD in which SES explains a significant portion of the association between Hispanic ethnicity and worse health outcomes. ${ }^{35}$ However, controlling for SES in our analysis, there were still significant differences in $\mathrm{MB}$ volumes and functional outcomes in the SVHD group compared to that in controls, and SES did not contribute to those variables. However, other important factors of SES such as maternal age and education may provide more of an impact than annual salary, as measured in this study.

Lastly, targeted interventions to improve memory are needed to mitigate these deficits in SVHD. Over the years, clinical management changes have been implemented to address delayed brain maturation by allowing the SVHD fetus to remain in utero for the entire 40-week gestation period, ${ }^{36}$ avoidance of hemodilution (hematocrit <25\%) during cardiopulmonary bypass, ${ }^{37}$ regional low-flow cerebral perfusion to increase cerebral perfusion during stage one palliation, ${ }^{38,39}$ and vigilant single ventricle interstage follow-up to optimize nutrition and growth. ${ }^{40}$ While these interventions individually have some modest benefit, many are not sufficient to fully alleviate the life-long cognitive outcomes in SVHD patients, and other interventions are needed to focus on ways to promote neurogenesis or regrowth of MBs to optimize memory processes and subsequent academic achievement and ability for self-care.

Table 2. Partial correlations between mammillary body volumes and cognitive scores in combined SVHD and controls

\begin{tabular}{|c|c|c|c|c|}
\hline \multirow{2}{*}{$\frac{\text { Variables }}{\text { WRAML2 GMI }}$} & \multicolumn{2}{|c|}{ Left mammillary body volume } & \multicolumn{2}{|c|}{ Right mammillary body volume } \\
\hline & $r=0.23$ & $p=0.07$ & $r=0.22$ & $p=0.09$ \\
\hline Verbal Memory Index (GMI) & $r=0.26$ & $p=0.04$ & $r=0.25$ & $p=0.06$ \\
\hline Visual Memory Index (GMI) & $r=0.13$ & $p=0.34$ & $r=0.20$ & $p=0.12$ \\
\hline Attention/Concentration (GMI) & $r=0.16$ & $p=0.22$ & $r=0.12$ & $p=0.37$ \\
\hline WRAML2 GRI & $r=0.18(n=62)$ & $p=0.17$ & $r=0.19(n=62)$ & $p=0.16$ \\
\hline Working Memory Index (GRI) & $r=0.04$ & $p=0.75$ & $r=0.04$ & $p=0.79$ \\
\hline Verbal Recognition Index (GRI) & $r=0.05(n=62)$ & $p=0.71$ & $r=0.10(n=62)$ & $p=0.47$ \\
\hline Visual Recognition Index (GRI) & $r=-0.02$ & $p=0.91$ & $r=-0.06$ & $p=0.64$ \\
\hline MoCA Total & $r=0.28$ & $p=0.03$ & $r=0.26$ & $p=0.04$ \\
\hline Visuospatial/Executive Function (MoCA) & $r=0.20$ & $p=0.13$ & $r=0.24$ & $p=0.07$ \\
\hline Naming (MoCA) & $r=0.02$ & $p=0.90$ & $r=0.05$ & $p=0.70$ \\
\hline Attention (MoCA) & $r=0.13$ & $p=0.32$ & $r=0.16$ & $p=0.23$ \\
\hline Language (MoCA) & $r=0.13$ & $p=0.33$ & $r=0.14$ & $p=0.30$ \\
\hline Abstraction (MoCA) & $r=0.16$ & $p=0.22$ & $r=0.19$ & $p=0.14$ \\
\hline Delayed Memory Recall (MoCA) & $r=0.34$ & $p=0.008$ & $r=0.29$ & $p=0.03$ \\
\hline Orientation (MoCA) & $r=-0.11$ & $p=0.42$ & $r=-0.13$ & $p=0.31$ \\
\hline
\end{tabular}

Covariates: age, sex, and TBV

MoCA Montreal Cognitive Assessment, GMI General Memory Index, WRAML2 Wide Range Assessment of Memory and Learning, Second Edition 
Table 3. Mammillary body volume in relation to cognitive outcomes for SVHD and controls

\begin{tabular}{|c|c|c|c|c|c|c|}
\hline Variables & \multicolumn{3}{|c|}{ Left mammillary body volume } & \multicolumn{3}{|c|}{ Right mammillary body volume } \\
\hline WRAML2 GMI & 0.29 & 0.22 & 0.07 & 0.29 & 0.23 & 0.09 \\
\hline Verbal Memory Index (GMI) & 0.18 & 0.28 & 0.04 & 0.17 & 0.27 & 0.06 \\
\hline Visual Memory Index (GMI) & 0.13 & 0.13 & 0.34 & 0.15 & 0.22 & 0.12 \\
\hline Attention/Concentration (GMI) & 0.19 & 0.16 & 0.22 & 0.18 & 0.13 & 0.37 \\
\hline Verbal Recognition Index (GRI) & 0.02 & 0.06 & 0.71 & 0.26 & 0.11 & 0.47 \\
\hline Visual Recognition Index (GRI) & 0.11 & -0.02 & 0.91 & 0.12 & -0.07 & 0.64 \\
\hline MoCA Total & 0.45 & 0.24 & 0.03 & 0.44 & 0.24 & 0.04 \\
\hline Visuospatial/executive function (MoCA) & 0.38 & 0.18 & 0.13 & 0.39 & 0.23 & 0.05 \\
\hline Naming (MoCA) & 0.06 & 0.02 & 0.90 & 0.06 & 0.06 & 0.70 \\
\hline Delayed memory recall (MoCA) & 0.23 & 0.35 & 0.008 & 0.20 & 0.32 & 0.03 \\
\hline Orientation (MoCA) & 0.01 & -0.12 & 0.42 & 0.02 & -0.16 & 0.31 \\
\hline
\end{tabular}

Linear regression analyses (dependent variable: functional outcomes). Variables included left and right mammillary body volume, total brain volume, sex, and age

WRAML2 Wide Range Assessment of Memory and Learning, Second Edition, GRI General Recognition Index, GMI General Memory Index, MoCA Montreal Cognitive Assessment

\section{Limitations}

Despite the small sample size, significant differences in $M B$ volumes emerged between groups. While the extensive exclusion criteria made a more homogeneous SVHD sample (gestation $<37$ weeks, no previous stroke, ECMO use, cardiac arrest, pacemaker, protein-losing enteropathy), this may reflect a group with better health within their chronic condition and thus may not be generalizable to all SVHD participants. Secondary to a small sample size, we combined SVHD and control participants to examine correlations. Further studies are required to assess such correlations in SVHD participants only. Overall Intelligence Quotient (IQ) was not measured as part of this study and we are unable to discern whether the memory impairment is isolated or part of general IQ reduction. In addition, there is the potential for subjective bias related to manual ROI tracings compared to automated programs. However, manual tracing is considered the gold standard due to the small size of the structures and close proximity to related structures. Lastly, without fetal or neonatal brain MRI, we are unable to specify the evolution of altered brain development leading to smaller $\mathrm{MB}$ volumes. In addition, we examined adolescents with SVHD at one time point representing the cumulative injury and are unable to determine the timing and mechanism of altered MB volumes in the condition.

\section{Conclusions}

Adolescents with SVHD who have undergone the Fontan procedure show significantly reduced left and right MB volumes, which may contribute to cognitive deficits. The mechanisms contributing to volume loss are likely multifactorial, including innate, nutritional, hypoxic/ischemic-induced processes, impaired $\mathrm{CBF}$, and potential preoperative and postoperative hemodynamic instability. Further investigation is needed to explore possible interventions to improve memory/cognition and promote neuroprotection, which could potentially enhance academic achievement, self-care, and quality of life in this high-risk population.

\section{ACKNOWLEDGEMENTS}

Authors thank Dr. Marie Poulsen, Chief Clinical Psychologist, Children's Hospital Los Angeles, Ms. Patty Chung, Mr. Luke Ehlert, Ms. Paola Moreno, and Ms. Madeline Townsley for assistance with data collection. This research and N.A.P., M.A.W., N.J.H., A.B.L., and R.K. received support by grants from the National Institutes of Health R01NR 013930 and R01NR016463.

\section{AUTHOR CONTRIBUTIONS}

All authors contributed to the conception and design of the study. C.C.-M.: mammillary body tracings, data collection and interpretation, draft manuscript, revisions, and final approval; B.R.: MRI data collection, processing, statistical analyses, draft review, and final manuscript approval; M.A.W.: draft review and final manuscript approval; S.S.: MRI data collection, processing, draft review, and final manuscript approval; S.M.: mammillary body tracings, draft review, and final manuscript approval; N.J.H and A.B.L.: participant recruitment, draft review, revisions, and final manuscript approval. R.K.: data interpretation, draft review, and final manuscript approval; N.A.P.: participant recruitment, data collection and interpretation, draft review, revisions, and final manuscript approval.

\section{ADDITIONAL INFORMATION}

Competing interests: The authors declare no competing interests.

Publisher's note Springer Nature remains neutral with regard to jurisdictional claims in published maps and institutional affiliations.

\section{REFERENCES}

1. Bellinger, D. C. et al. Neuropsychological status and structural brain imaging in adolescents with single ventricle who underwent the Fontan procedure. J. Am. Heart Assoc. 4, e002302 (2015).

2. Marelli, A. J., Mackie, A. S., lonescu-Ittu, R., Rahme, E. \& Pilote, L. Congenital heart disease in the general population: changing prevalence and age distribution. Circulation 115, 163-172 (2007).

3. Gaynor, J. W. et al. Neurodevelopmental outcomes after cardiac surgery in infancy. Pediatrics 135, 816-825 (2015). 
4. Atz A. M., et al. Longitudinal outcomes of patients with single ventricle after the Fontan procedure. J. Am. Coll. Cardiol. 69, 2735-2744 (2017).

5. Pike, N. A. et al. Quality of life, health status, and depression: comparison between adolescents and adults after the Fontan procedure with healthy counterparts. $J$. Cardiovasc. Nurs. 27, 539-546 (2012).

6. Singh, $\mathrm{S}$. et al. Regional brain gray matter changes in adolescents with single ventricle heart disease. Neurosci. Lett. 665, 156-162 (2018).

7. Munoz-Lopez, M. et al. Hippocampal damage and memory impairment in congenital cyanotic heart disease. Hippocampus 27, 417-424 (2017).

8. Vann, S. D. Re-evaluating the role of the mammillary bodies in memory. Neuropsychologia 48, 2316-2327 (2010).

9. Kumar, R. et al. Reduced mammillary body volume in patients with obstructive sleep apnea. Neurosci. Lett. 438, 330-334 (2008).

10. Kumar, R. et al. Mammillary bodies and fornix fibers are injured in heart failure Neurobiol. Dis. 33, 236-242 (2009).

11. Kumar, R., Lee, K., Macey, P. M., Woo, M. A. \& Harper, R. M. Mammillary body and fornix injury in congenital central hypoventilation syndrome. Pediatr. Res. 66, 429-434 (2009).

12. van Asselen, M., Kessels, R. P., Wester, A. J. \& Postma, A. Spatial working memory and contextual cueing in patients with Korsakoff amnesia. J. Clin. Exp. Neuropsychol. 27, 645-655 (2005).

13. Sheslow, D. \& Adams, W. Wide Range Assessment of Memory and Learning (WRAML2), 2nd edn (Wide Range Inc., Delaware, 2003).

14. Pike, N. A., Poulsen, M. K. \& Woo, M. A. Validity of the Montreal Cognitive Assessment screener in adolescents and young adults with and without congenital heart disease. Nurs. Res. 66, 222-230 (2017).

15. Pike, N. A. et al. Predictors of memory deficits in adolescents and young adults with congenital heart disease compared to healthy controls. Front. Pediatr. 4, 117 (2016).

16. Nasreddine, Z. S. et al. The Montreal Cognitive Assessment, MoCA: a brief screening tool for mild cognitive impairment. J. Am. Geriatr. Soc. 53, 695-699 (2005).

17. Ashburner, J. \& Friston, K. J. Unified segmentation. Neuroimage 26, 839-851 (2005).

18. Miller, S. P. et al. Abnormal brain development in newborns with congenital heart disease. N. Engl. J. Med. 357, 1928-1938 (2007).

19. Rollins, C. K. et al. White matter microstructure and cognition in adolescents with congenital heart disease. J. Pediatr. 165, e931-e932 (2014).

20. Nagaraj, U. D. et al. Impaired global and regional cerebral perfusion in newborns with complex congenital heart disease. J. Pediatr. 167, 1018-1024 (2015).

21. Sun, L. et al. Reduced fetal cerebral oxygen consumption is associated with smaller brain size in fetuses with congenital heart disease. Circulation 131, 1313-1323 (2015).

22. Andropoulos, D. B. et al. Brain immaturity is associated with brain injury before and after neonatal cardiac surgery with high-flow bypass and cerebral oxygenation monitoring. J. Thorac. Cardiovasc. Surg. 139, 543-556 (2010).

23. Mahle, W. T., Clancy, R. R., McGaurn, S. P., Goin, J. E. \& Clark, B. J. Impact of prenatal diagnosis on survival and early neurologic morbidity in neonates with the hypoplastic left heart syndrome. Pediatrics 107, 1277-1282 (2001).
24. Santin, L. J., Rubio, S., Begega, A. \& Arias, J. L. Effects of mammillary body lesions on spatial reference and working memory tasks. Behav. Brain Res. 102, 137-150 (1999).

25. Kelleher, D. K., Laussen, P., Teixeira-Pinto, A. \& Duggan, C. Growth and correlates of nutritional status among infants with hypoplastic left heart syndrome (HLHS) after stage 1 Norwood procedure. Nutrition 22, 237-244 (2006).

26. Goldberg, C. S. et al. Factors associated with neurodevelopment for children with single ventricle lesions. J. Pediatr. 165, 490.e8-496.e8 (2014).

27. Williams, R. V. et al. Birth weight and prematurity in infants with single ventricle physiology: pediatric heart network infant single ventricle trial screened population. Congenit. Heart Dis. 5, 96-103 (2010).

28. Ono, M. et al. Somatic development long after the Fontan operation: factors influencing catch-up growth. J. Thorac. Cardiovasc. Surg. 134, 1199-1206 (2007).

29. Pike, N. A. et al. Sex and age differences in body-image, self-esteem, and body mass index in adolescents and adults after single-ventricle palliation. Pediatr. Cardiol. 33, 705-712 (2012).

30. Bilici, R. et al. 2015 A case of Wernicke-Korsakoff syndrome treated 1 year after the onset of symptoms. Prim. Care Companion CNS Disord. 17, 10.4088/ PCC.15I01801 (2015).

31. Langlais, P. J. \& Savage, L. M. Thiamine deficiency in rats produces cognitive and memory deficits on spatial tasks that correlate with tissue loss in diencephalon, cortex and white matter. Behav. Brain Res. 68, 75-89 (1995).

32. Khalsa, S. S., Kumar, R., Patel, V., Strober, M. \& Feusner, J. D. Mammillary body volume abnormalities in anorexia nervosa. Int. J. Eat. Disord. 49, 920-929 (2016).

33. Harper, C. Thiamine (vitamin B1) deficiency and associated brain damage is still common throughout the world and prevention is simple and safe! Eur. J. Neurol. 13, 1078-1082.34 (2006).

34. Shamir, R. et al. Thiamine deficiency in children with congenital heart disease before and after corrective surgery. JPEN J. Parenter. Enter. Nutr. 24, 154-158 (2000).

35. Peyvandi, S. et al. Socioeconomic mediators of racial and ethnici disparities in congenital heart disease outcomes: a population-based study in California. J. Am. Heart Assoc. 7, e010342 (2018).

36. Ohye, R. G., Schranz, D. \& D'Udekem, Y. Current therapy for hypoplastic left heart syndrome and related single ventricle lesions. Circulation 134, 1265-1279 (2016).

37. Wypij, D. et al. The effect of hematocrit during hypothermic cardiopulmonary bypass in infant heart surgery: results from the combined Boston hematocrit trials. J. Thorac. Cardiovasc. Surg. 135, 355-360 (2008).

38. Chock, V. Y. et al. Antegrade cerebral perfusion reduces apoptotic neuronal injury in a neonatal piglet model of cardiopulmonary bypass. J. Thorac. Cardiovasc. Surg. 131, 659-665 (2006).

39. Dent, C. L. et al. Brain magnetic resonance imaging abnormalities after the Norwood procedure using regional cerebral perfusion. J. Thorac. Cardiovasc. Surg. 131, 190-197 (2006).

40. Di Maria, M. V. et al. Supplemental tube feeding does not mitigate weight loss in infants with shunt-dependent single-ventricle physiology. Pediatr. Cardiol. 34 1350-1356 (2013). 